\title{
Results for light pseudoscalar mesons
}

\author{
A. Bazavov, W. Freeman and D. Toussaint \\ Department of Physics, University of Arizona, Tucson, AZ 85721, USA \\ C. Bernard* and X. Du \\ Department of Physics, Washington University, St. Louis, MO 63130, USA \\ E-mail: cbewust 1 . edu

\section{DeTar, L. Levkova and M.B. Oktay} \\ Physics Department, University of Utah, Salt Lake City, UT 84112, USA

\section{Steven Gottlieb} \\ Department of Physics, Indiana University, Bloomington, IN 47405, USA \\ and NCSA, University of Illinois, Urbana, IL 61801, USA
}

\section{Urs M. Heller}

American Physical Society, One Research Road, Ridge, NY 11961, USA

\section{J.E. Hetrick}

Physics Department, University of the Pacific, Stockton, CA 95211, USA

\section{J. Laiho}

SUPA, School of Physics \& Astronomy, University of Glasgow, Glasgow G12 8QQ, UK

\section{J. Osborn}

Argonne Leadership Computing Facility, Argonne National Laboratory, Argonne, IL 60439, USA

\section{R. Sugar}

Department of Physics, University of California, Santa Barbara, CA 93106, USA

\section{R.S. Van de Water}

Department of Physics, Brookhaven National Laboratory, Upton, NY 11973, USA

We present the current status of the MILC collaboration's calculations of the properties of the light pseudoscalar meson sector. We use asqtad staggered ensembles with $2+1$ dynamical flavors down to $a \approx 0.045 \mathrm{fm}$ and light quark mass down to $0.05 m_{s}$. Here we describe fits to the data using chiral forms from SU(3) chiral perturbation theory, including all staggered taste violations at NLO and the continuum NNLO chiral logarithms. We emphasize issues of convergence of the chiral expansion.

The XXVIII International Symposium on Lattice Field Theory, Lattice2010

June 14-19, 2010

Villasimius, Italy

\footnotetext{
*Speaker.
} 


\section{Introduction}

The MILC collaboration has been carrying out simulations of $2+1$ flavor lattice QCD with the improved "asqtad" staggered quark action since 1999; for a review of the physics program see Ref. [1]. The asqtad ensembles are now complete, and we are progressing to lattice generation with the more highly improved HISQ action [2], with promising results so far [3]. Extraction of physics with the asqtad ensembles is however continuing. In particular, we are in the process of finishing our (asqtad) study of the light pseudoscalar meson sector. Here we give the latest update of this project, focusing on $\mathrm{SU}(3)$ chiral fits.

Compared to the last status reports in Refs. [4, 5], we have completed the final four ensembles, adding between 35 and 100\% more configurations. The ensembles include two of the ones with lighter-than-physical strange quark masses, which are crucial for $\mathrm{SU}(3)$ chiral fits. This new data has allowed us to remove all a priori restrictions on SU(3) NNLO low energy constants (LECs) from the fits. It is therefore now possible, for the first time, to report physical values for some of these NNLO LECs, although the errors in the values are quite large. In addition, we now consider additional alternative versions of the chiral fits, including different treatments of still higher order effects. These changes in the analysis give somewhat larger systematic errors on the LECs at NLO, as well as on the decay constant and quark condensate in the three-flavor chiral limit. On the other hand, errors on $f_{\pi}$ and $f_{K}$, and on quark masses, are largely insensitive to the changes, and indeed have continued to decrease as the ensembles have improved.

We have also examined in detail the convergence of the $\mathrm{SU}(3)$ chiral expansion, as a function of quark mass. Results are presented both for the case of three degenerate quark masses and for the more physical case of one quark (strange) much heavier than the other two. The expansion appears to be significantly better behaved in the former case than in the latter one.

\section{The ensembles and the fitting procedures}

The present analysis uses MILC ensembles at $a \approx 0.09 \mathrm{fm}, a \approx 0.06 \mathrm{fm}$ and $a \approx 0.045 \mathrm{fm}$. Although the MILC collaboration has also generated ensembles at three coarser lattice spacings from $a \approx 0.18 \mathrm{fm}$ to $a \approx 0.12 \mathrm{fm}$, using that data in the fits is not currently feasible, since it would require analytic control of higher order discretization effects. The ensembles used in this study are listed in Table 1 . The quantity $\hat{m}^{\prime}$ is the simulated light $(u, d)$ quark mass. Three ensembles have an unphysically light simulated strange quark mass $m_{s}^{\prime} \approx 0.6 m_{s}$ (two $a \approx 0.09$ ensembles with $a m_{s}^{\prime}=0.0186$, and one $a \approx 0.06$ ensemble with $a m_{s}^{\prime}=0.0108$ ), and one ensemble has three degenerate quarks with $\hat{m}^{\prime}=m_{s}^{\prime} \approx 0.1 m_{s}$ (the $a \approx 0.09$ ensemble with $a \hat{m}^{\prime}=a m_{s}^{\prime}=0.0031$ ). These light- $m_{s}^{\prime}$ ensembles were created specifically to help control the SU(3) $\chi \mathrm{PT}$ fits.

As always in MILC staggered simulations, we take the fourth root of the fermion determinant in order to eliminate the effect of unwanted degrees of freedom ("tastes") in the lattice generation. Recent work (see Ref. [1] and [6] for reviews) makes a strong case that the procedure does indeed produce the desired theory in the continuum limit.

At the fairly small lattice spacings considered here, chiral effects due to taste-violations are relatively small but are nevertheless not negligible. We take these effects into account at NLO in our chiral fits by using rooted staggered $\chi \mathrm{PT}$ (rS $\chi \mathrm{PT}$ ) [7]. An rS $\chi$ PT calculation of the two-loop chiral 


\begin{tabular}{|c|c|c|c|c|c|c|c|}
\hline$a(\mathrm{fm})$ & $a \hat{m}^{\prime} / a m_{s}^{\prime}$ & $10 / g^{2}$ & size & \# lats. & $u_{0}$ & $r_{1} / a$ & $m_{\pi} L$ \\
\hline$\approx 0.09$ & $0.0124 / 0.031$ & 7.11 & $28^{3} \times 96$ & 531 & 0.8788 & 3.858 & 5.78 \\
$\approx 0.09$ & $0.0093 / 0.031$ & 7.10 & $28^{3} \times 96$ & 1124 & 0.8785 & 3.823 & 5.04 \\
$\approx 0.09$ & $0.0062 / 0.031$ & 7.09 & $28^{3} \times 96$ & 591 & 0.8782 & 3.789 & 4.14 \\
$\approx 0.09$ & $0.00465 / 0.031$ & 7.085 & $32^{3} \times 96$ & $984^{*}$ & 0.8781 & 3.772 & 4.11 \\
$\approx 0.09$ & $0.0031 / 0.031$ & 7.08 & $40^{3} \times 96$ & 945 & 0.8779 & 3.755 & 4.21 \\
$\approx 0.09$ & $0.00155 / 0.031$ & 7.075 & $64^{3} \times 96$ & $751^{*}$ & 0.877805 & 3.738 & 4.80 \\
\hline$\approx 0.09$ & $0.0062 / 0.0186$ & 7.10 & $28^{3} \times 96$ & 985 & 0.8785 & 3.8823 & 4.09 \\
$\approx 0.09$ & $0.0031 / 0.0186$ & 7.06 & $40^{3} \times 96$ & $781^{*}$ & 0.8774 & 3.687 & 4.22 \\
$\approx 0.09$ & $0.0031 / 0.0031$ & 7.045 & $40^{3} \times 96$ & $555^{*}$ & 0.8770 & 3.637 & 4.20 \\
\hline$\approx 0.06$ & $0.0072 / 0.018$ & 7.48 & $48^{3} \times 144$ & 594 & 0.8881 & 5.399 & 6.33 \\
$\approx 0.06$ & $0.0054 / 0.018$ & 7.475 & $48^{3} \times 144$ & 465 & 0.88800 & 5.376 & 5.48 \\
$\approx 0.06$ & $0.0036 / 0.018$ & 7.47 & $48^{3} \times 144$ & 751 & 0.88788 & 5.353 & 4.49 \\
$\approx 0.06$ & $0.0025 / 0.018$ & 7.465 & $56^{3} \times 144$ & 768 & 0.88776 & 5.330 & 4.39 \\
$\approx 0.06$ & $0.0018 / 0.018$ & 7.46 & $64^{3} \times 144$ & 826 & 0.88764 & 5.307 & 4.27 \\
$\approx \approx 0.06$ & $0.0036 / 0.0108$ & 7.46 & $64^{3} \times 144$ & 601 & 0.88765 & 5.307 & 5.96 \\
\hline$\approx 0.045$ & $0.0028 / 0.014$ & 7.81 & $64^{3} \times 192$ & 801 & 0.89511 & 7.208 & 4.56 \\
\hline
\end{tabular}

Table 1: List of ensembles used in this study, with $u_{0}$ the tadpole factor and $r_{1} / a$ the scale from the heavy quark potential. The $r_{1} / a$ values are "mass-independent," obtained by using an interpolating fit to adjust the sea quark masses to their physical values [1]. Errors in these $r_{1} / a$ values are roughly 3 to 10 in the last digit. Configuration numbers with asterisks have been updated since last year.

logarithms does not exist, so at NNLO we use the continuum partially quenched chiral logarithms [8], with the the root mean square taste average of the pion mass as argument. In what we call "low-mass" fits (using the low- $m_{s}^{\prime}$ ensembles, with low valence masses), neglect of taste-violating effects is justified at NNLO because such effects are smaller than the mass effects that we keep. Thus such NNLO low-mass fits are "systematic" in the sense of $\chi$ PT. See Ref. [4] for more details.

In Ref. [4], the low-mass fits used prior widths to constrain the NNLO LECs to be $\mathscr{O}(1)$ in natural units [9]; LO and NLO LECs were unconstrained. With the current complete data set, we can dispense with the constraints and still obtain convergent fits. The data alone now determines the NNLO LECs. It is therefore possible to quote physical results for these quantities, although, not surprisingly, the errors at NNLO are quite large. Eliminating constraints at NNLO also tends to increase the errors of the NLO LECs, because there can be more trade-off between the two orders in the fit. Clearly the new errors are more conservative. Fortunately, eliminating constraints has very little effect on quantities extrapolated to physical meson masses, such as the quark masses and the decay constants $f_{\pi}$ and $f_{K}$. Such quantities are determined largely by the lattice data (which now goes to quite low light quark masses) and are not very sensitive to how the fits divide up contributions by orders in $\chi \mathrm{PT}$.

Another feature of the current analysis also tends to increase, relative to Ref. [4], our estimate of errors of certain quantities, in particular, the values of quantities in the 3-flavor chiral limit. Define the chiral coupling relevant at two-loops as $1 /\left(16 \pi^{2} f_{N N L O}^{2}\right)$. The value of the decay constant $f_{N N L O}$ in the coupling is ambiguous a priori because differences in $f_{N N L O}$ (between, say, the 3flavor chiral-limit value, $f_{3}$, and the physical $f_{\pi}$ ) are higher order. In Refs. [9, 1], which did not 

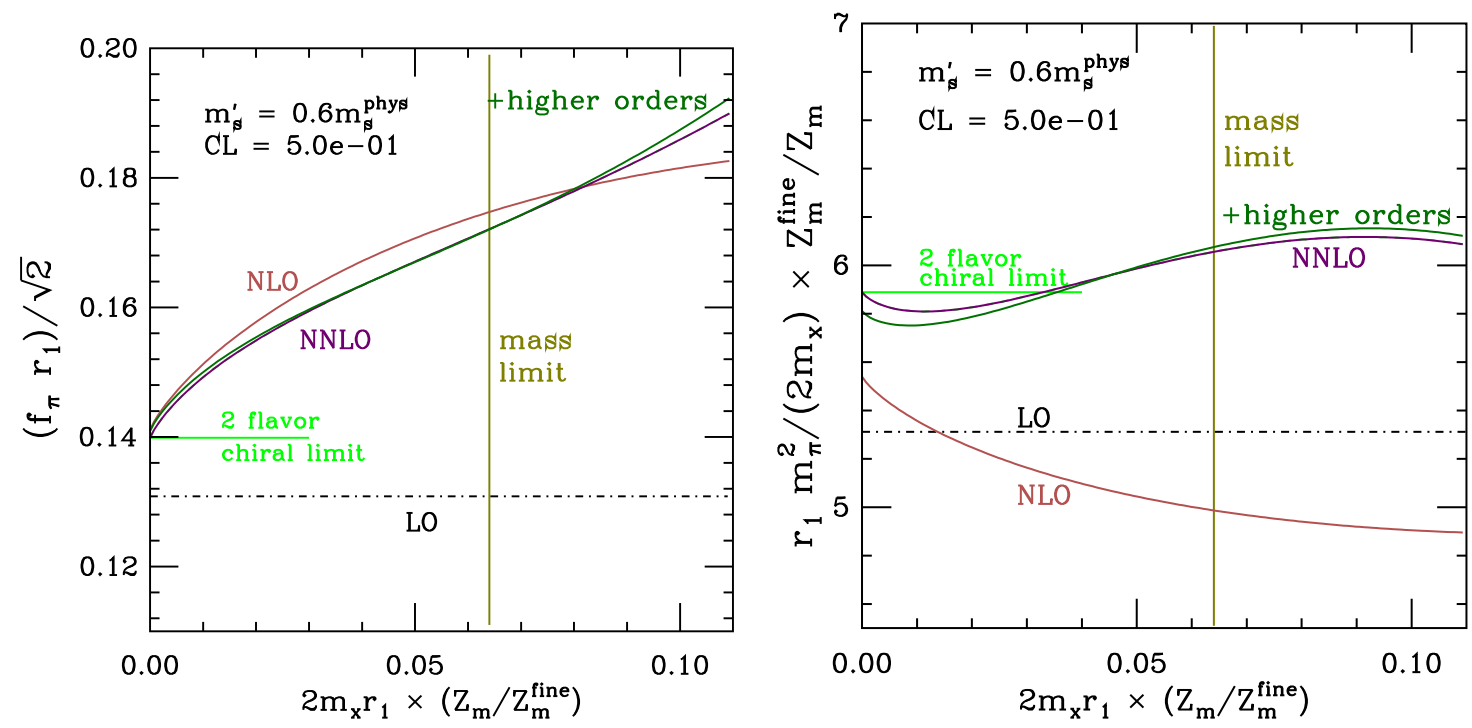

Figure 1: Convergence of "type-A" NNLO low-mass SU(3) chiral fits for $f_{\pi}$ (left) and $m_{\pi}^{2} / 2 m_{x}$ (right) as a function of the (degenerate) valence quark mass $m_{x}$. The strange sea quark mass $m_{s}^{\prime}$ is held fixed at $\approx 0.6 m_{s}$. The lines labeled LO, NLO, and NNLO show the $\chi$ PT contribution up to and including the indicated order. The line labeled "+higher orders" shows the effect of adding still higher order analytic terms. The vertical line labeled "mass limit" corresponds to the largest value of the valence mass (in the units of the abscissa) used in the fits, and is given by $2 m_{x} \approx m_{s}^{\prime}$. The fit has been extrapolated to the continuum.

include NNLO chiral logs, the corresponding issue already arose at NLO. We found there that using the "bare" coupling with $f_{N L O}=f_{3}$ did not give acceptable fits, and it was necessary to use a "physical" NLO coupling (with $f_{\pi} \lesssim f_{N L O} \lesssim f_{K}$ ) to get good fits to our lattice data. Similarly, here we cannot obtain good fits of our low-mass data with $f_{N N L O}=f_{3}$ : The best such fits have confidence level $\mathrm{CL}<0.03$, and in addition require unreasonably large lattice-spacing dependence. On the other hand, using $f_{N N L O} \approx f_{\pi}$ gives good fits, and indeed a value close to $f_{\pi}$ is chosen by the data if $f_{N N L O}$ in the NNLO terms is allowed to be a free parameter.

Fixing the value of $f_{N N L O}$ is done in two different ways in our fits. Our standard approach ("type-A fits"), used also in Ref. [4], is to put, for each ensemble, $f_{N N L O}=\tau f_{3}^{\text {fit }}$, where $f_{3}^{\text {fit }}$ is the decay constant in the chiral limit on that ensemble, and $\tau$ is a fixed number. The value of $\tau$ is chosen (iteratively) so that $f_{N N L O}$ has the desired value in the continuum limit. For the central value fits we ensure $f \cong f_{\pi}$ in the continuum, and other fits vary $f_{N N L O}$ over a range (roughly $0.95 f_{\pi} \lesssim f_{N N L O} \lesssim 1.15 f_{\pi}$ ) that gives acceptable CLs. An alternative approach ("type-B fits") that we have tried recently is simply to set, on all ensembles, $f_{N N L O}=\rho f_{\pi}$, where $f_{\pi}$ is taken from experiment, and $\rho$ is a fixed number, again chosen over the range that gives acceptable fits. The type-B approach completely decouples the value of $f_{N N L O}$ from the chiral limit quantity $f_{3}^{\text {fit }}$ that appears at lower order and should describe the decay constant in the low-mass regime.

Chiral fitting is then done in two stages. Systematic low-mass fits are performed first and are used to determine low energy constants (LECs) through NNLO: LO parameters $f_{3}$ and $B_{3}$ (often called $F_{0} \sqrt{2}$ and $B_{0}$ in the literature), NLO Gasser-Leutwyler [10] parameters $L_{i}$, and NNLO parameters $[11,8] K_{i}$ (partially quenched) or $C_{i}$ (unquenched $\mathrm{SU}(3)$ ). The fits include all partially quenched data for pion and kaon (with lighter than physical strange quark mass) decay constants and masses (108 points) and have 31 parameters. The full covariance matrix is used, and the CL is 

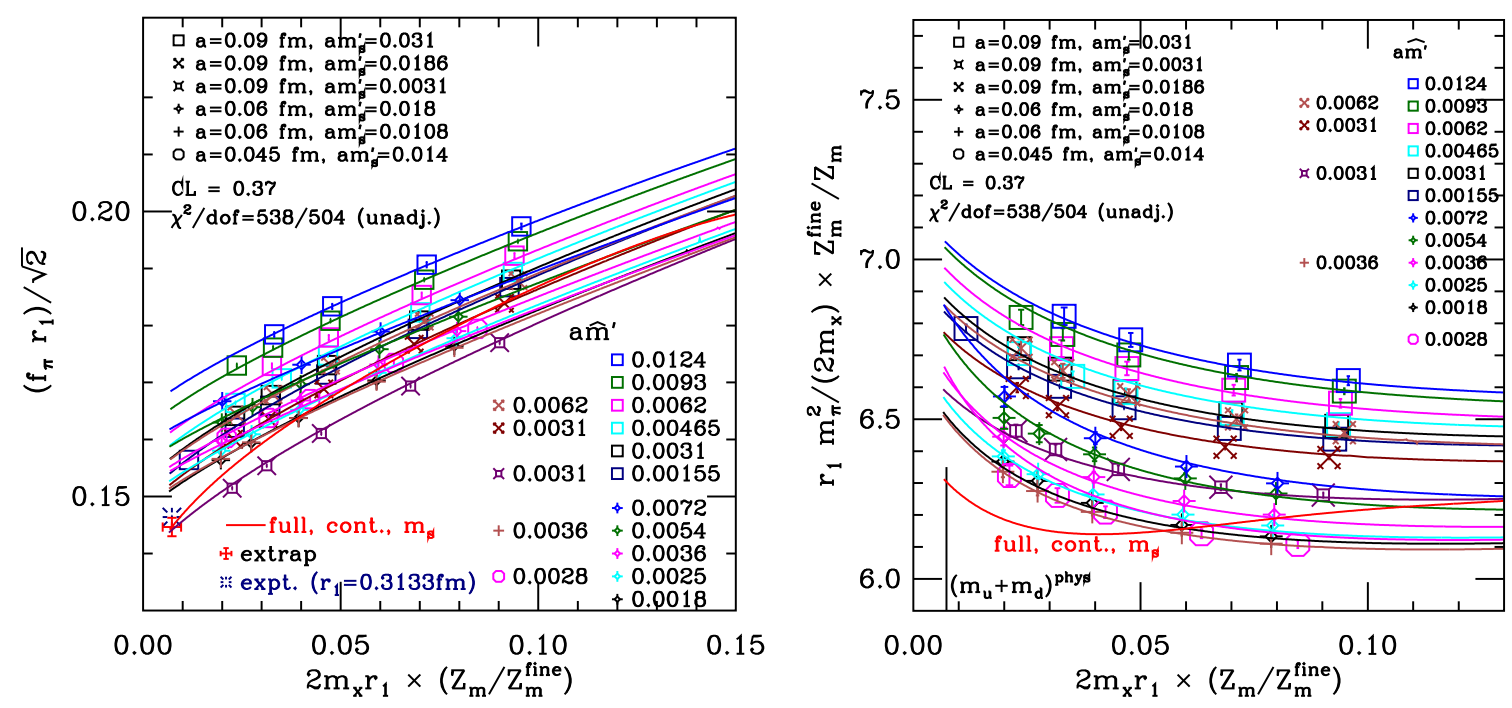

Figure 2: High-mass, type-A, $\mathrm{SU}(3)$ chiral fits, using $r_{1}=0.3133$. We show partially quenched data points with degenerate valence masses $m_{x}$ for the decay constant (left) and for $m_{\pi}^{2} /\left(2 m_{x}\right)$ (right). The red lines show the results after extrapolation to the continuum, putting $m_{s}^{\prime}=m_{s}$, and setting light sea and valence masses equal. The decay constant is also extrapolated to the physical mass point and compared with experiment (blue burst).

good (0.50). The convergence of $\chi \mathrm{PT}$ in these fits (type-A) is shown in Fig. 1. Although the NLO terms for $m_{\pi}^{2} / 2 m_{x}$ seem to be anomalously small, the overall convergence is satisfactory.

In the second stage of fitting, the "high-mass" fits, all ensembles listed in Table 1 are included with the valence masses restricted to $m_{x}+m_{y} \leq 1.2 m_{s}$. In the type-A case, the LO, NLO, NNLO LECs are fixed (for central values) or fixed within statistical errors at the values from the low-mass fits. $\mathrm{N}^{3} \mathrm{LO}$ and $\mathrm{N}^{4} \mathrm{LO}$ analytic terms are included, but not the corresponding logs. These terms are needed to obtain good CLs, and they allow us to interpolate around the (physical) strange quark mass. The fact that they are required indicates that SU(3) $\chi \mathrm{PT}$ is not converging well at these mass values, unlike the situation in the low-mass case (see below for more evidence). However, since the LO, NLO, and NNLO LECs dominate the chiral extrapolation to the physical point, the results for decay constants and masses are rather insensitive to the form of these $\mathrm{N}^{3} \mathrm{LO}$ and $\mathrm{N}^{4} \mathrm{LO}$ interpolating terms, as long as the fits are good.

In the type-B case, high-mass fits with the LO, NLO, and NNLO LECs fixed at their low-mass values do not give good CLs. We can obtain acceptable fits by allowing these LECs to vary, with prior widths set by the low-mass statistical errors. But in that case, several of the LECs move by more than $2 \sigma$. For these reasons the type-B fits are currently disfavored, although we do include them as alternative chiral fits in estimating systematic errors.

The (central value, type-A) high-mass fits are illustrated for pions (degenerate valence points) in Fig. 2, where the scale has been set by $r_{1}=0.3133(23) \mathrm{fm} \mathrm{[12].} \mathrm{The} \mathrm{extrapolations} \mathrm{to} \mathrm{the}$ continuum and to the physical masses are also shown. The agreement of our result, $f_{\pi}=129.2 \pm$ $0.4 \pm 1.4 \mathrm{MeV}$, with the experimental value (130.4(2) MeV [13]) is good. From now on we set the scale using $f_{\pi}$, which gives $r_{1}=0.3106(8)(14)(4) \mathrm{fm}$, where the last error is experimental.

Figure 3 shows the convergence of $\chi \mathrm{PT}$ for the pion decay constant. The left plot is for the 

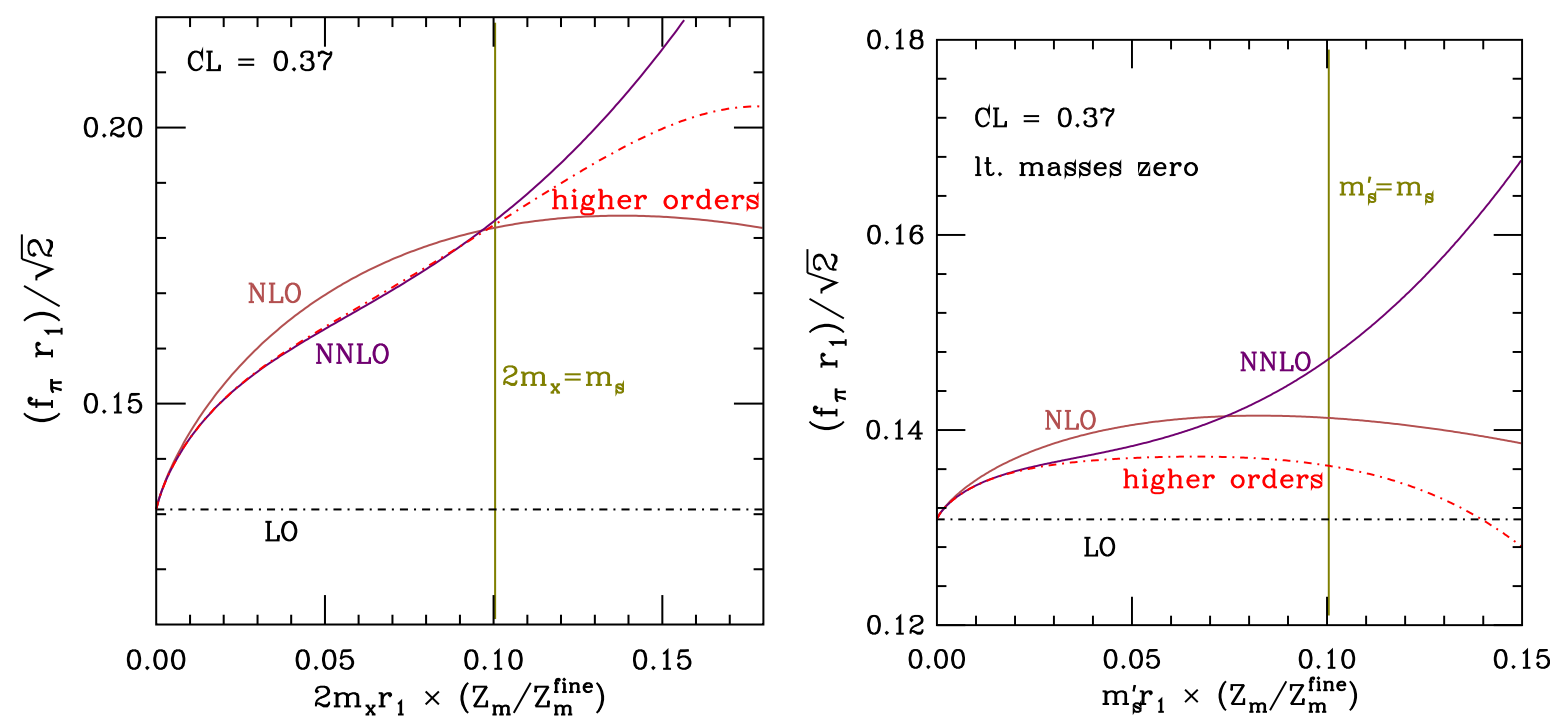

Figure 3: Convergence of $\chi$ PT for the decay constant, with the same fit as in Fig. 2. Left, the degenerate case $\left(m_{s}^{\prime}=\hat{m}^{\prime}=m_{x}\right)$ as a function of $m_{x}$. Right, 2-flavor chiral limit $m_{x}=\hat{m}^{\prime}=0$ as a function of $m_{s}^{\prime}$. These fits have been extrapolated to the continuum.

degenerate 3-flavor case. Note that the convergence is still good significantly beyond the point where $2 m_{x}=m_{s}$, where the meson mass is already close to the physical value of $m_{K}$. The right plot gives the decay constant as a function of the strange sea quark mass $m_{s}^{\prime}$, with the valence and light sea masses extrapolated to zero (the 2-flavor chiral limit). The vertical line indicates $m_{s}^{\prime}=m_{s}$, when the kaon is near physical. Here the convergence is reasonable only up to about $m_{s}^{\prime}=0.6 m_{s}$; by $m_{s}^{\prime}=0.8 m_{s}$ the NNLO term has the wrong sign, and moves the NLO result away from the "higher orders" line (which must be close to what the lattice data demands, since the fit is good). Nevertheless, the NLO contribution remains reasonable; the problem is mainly at NNLO. This kind of behavior is not unexpected for an asymptotic expansion.

For the $m_{\pi}^{2} /\left(2 m_{x}\right)$ case (not shown), the convergence of $\chi \mathrm{PT}$ is similar to that of the decay constant. Again, convergence in the degenerate 3-flavor case is good up to meson masses about $10 \%$ or $15 \%$ higher than the kaon. In the 2-flavor chiral limit, the behavior as a function of $m_{s}^{\prime}$ appears to be comparable to that of the decay constant, breaking down before $m_{s}^{\prime}=m_{s}$.

\section{Preliminary results}

The following is a sample of our current results:

$$
\begin{aligned}
f_{K}=156.1 \pm 0.4_{-0.9}^{+0.6} \mathrm{MeV}, & f_{K} / f_{\pi}=1.197(2)\left({ }_{-7}^{+3}\right), \\
V_{u s}=0.2247\left(\left(_{-9}^{+14}\right),\right. & f_{3}=113.6 \pm 3.6 \pm 7.7 \mathrm{MeV}, \\
f_{2}=123.0 \pm 0.5 \pm 0.7 \mathrm{MeV}, & 2 L_{8}-L_{5}=-0.51(11)\left({ }_{-19}^{+45}\right), \\
2 L_{6}-L_{4}=0.09(24)\left({ }_{-27}^{+32}\right), & L_{5}=1.79(16)\left({ }_{-41}^{+28}\right), \\
L_{4}=0.19(22)\left({ }_{-33}^{+57}\right), & 2 C_{21}-C_{19}=1.5(6)\left({ }_{-4}^{+6}\right), \\
K_{19}=3.5 \pm 1.2_{-0.7}^{+2.3}, & K_{39}-K_{17}=3.4 \pm 1.5_{-1.6}^{+1.8},
\end{aligned}
$$




$$
\bar{l}_{3}=3.18(50)(89), \quad \bar{l}_{4}=4.29(21)(82),
$$

where errors are statistical and systematic. $f_{2}$ is the decay constant in the 2-flavor chiral limit. The NLO LECs $L_{i}$ are in units of $10^{-3}$, and the NNLO LECs $K_{i}$ and $C_{i}$ are in units of $10^{-6}$; both are at chiral scale $m_{\eta}$. Other $K_{i}$ and $C_{i}$ are also of this order of magnitude, but most have statistical or systematic errors that are more than $100 \%$. The scale invariant SU(2) LECs $\bar{\ell}_{3,4}$ are obtained from the SU(3) LECs using the two-loop conversion formulae [14]. There is good agreement between the $\mathrm{SU}(3)$ chiral fit results described here and the results of the $\mathrm{SU}(2)$ chiral fits $[5,15]$ for all quantities that can be directly compared.

As discussed above, errors for $f_{3}$ and the NLO LECs are larger than previous ones [4], while those for $f_{K}, f_{K} / f_{\pi}, f_{2}$, as well as for quark masses (not given here), are similar to or somewhat smaller than before. In general, we use type-A fits with $f_{N N L O} \cong f_{\pi}$ for central values, and alternative chiral fits (including type-B) for systematic error estimates. The exception is $f_{3}$, where we have averaged type-A and type-B results and symmetrized the errors. This is because the distribution for $f_{3}$ from type-A and type-B fits is bimodal, with the former giving values toward the high end of the range, and the latter giving values toward the low end. The large uncertainty in the 3-flavor chiral limit, compared to the physical point, comes ultimately from the fact that we only have one usable ensemble with three degenerate light flavors, while there are many with $m_{s}^{\prime} \approx m_{s}$.

We thank J. Bijnens for his program to compute the partially quenched NNLO chiral logs.

\section{References}

[1] A. Bazavov et al., Rev. Mod. Phys. 82, 1349 (2010) [arXiv:0903.3598].

[2] E. Follana et al. [HPQCD Collaboration], Phys. Rev. D 75, 054502 (2007) [hep-lat/0610092].

[3] A. Bazavov et al. (MILC), Phys. Rev. D 82, 074501 (2010) [arXiv:1004.0342].

[4] A. Bazavov et al., PoS (LAT2009) 079 (2009) [arXiv:0910.3618] and PoS (CD 09 ) 007 (2009) [arXiv:0910.2966].

[5] A. Bazavov et al., PoS (LAT2009) 077 (2009) [arXiv:0911.0472].

[6] M. Golterman, PoS (CONF INEMENT8) 014 (2008) [arXiv:0812.3110].

[7] C. Aubin and C. Bernard, Phys. Rev. D 68, 034014 (2003) [hep-lat/0304014]; and 074011 (2003) [hep-lat/0306026].

[8] J. Bijnens, N. Danielsson and T.A. Lahde, Phys. Rev. D 70, 111503 (2004) [hep-lat/0406017] and 73, 074509 (2006) [hep-lat/0602003]; J. Bijnens and T.A. Lahde, Phys. Rev. D 71, 094502 (2005) [hep-lat/0501014].

[9] C. Aubin et al. (MILC), Phys. Rev. D 70, 114501 (2004) [hep-lat/0407028].

[10] J. Gasser and H. Leutwyler, Nucl. Phys. B 250, 465 (1985).

[11] J. Bijnens, G. Colangelo, G. Ecker, JHEP 9902, 020 (1999) [hep-ph/9902437].

[12] C. T. H. Davies et al. [ HPQCD Collaboration ], Phys. Rev. D 81, 034506 (2010). [arXiv:0910.1229].

[13] K. Nakamura et al. (Particle Data Group), J. Phys. G 37, 075021 (2010).

[14] J. Gasser, C. Haefeli, M. A. Ivanov and M. Schmid, Phys. Lett. B652, 21 (2007) [arXiv:0706.0955].

[15] X. Du et al., POS (LAT2010) (these proceedings). 